\title{
Ethical Issues in Biomedical Research and Publication
}

(BIRDEM Med J 2018; 8(1): 1-8)

"Ethics" in simple terms is defined as "norms for conduct" that distinguishes between acceptable and unacceptable behavior. Though ethical norms are learnt since childhood at home, school, religious places, etc., it is influenced throughout the life, impacted by various life experiences which explain the large variations in interpretation of ethical norms among individuals.

Ethical norms related to biomedical research and publication are laid down by various national and international agencies. The Nuremberg Code and Declaration of Helsinki by World Medial Association is the landmark in ethical standards followed all over the world for biomedical research and uniform requirements for manuscripts submitted to biomedical journals (formulated by International Committee of Medical Journal Editors) for publication.

The best evidence shows that misconduct is a very rare occurrence in research and there is no evidence that science has become ethically corrupt. The rate of misconduct has been estimated to be as low as $0.01 \%$ to as high as $1 \%$ of researchers per year. According to the "bad apple" theory, most scientists are highly ethical. Only those who are morally corrupt, economically desperate (in cases of grant application), or psychologically disturbed will commit misconduct.

Research and publication play a very vital role in progress of sciences in any field. As publication of scientific papers is now linked to professional career up gradation, the pressure for publication of papers is tremendous. Postgraduate teachers lack adequate experience themselves and time to guide the students into research design, its execution, analysis, and more importantly, into research norms and ethics. In current scenario, there is a real danger of compromising the scientific quality of research papers. We need to focuse on four general ethical issues: authorship, peer review, duplicate or repetitive publication, and conflict of interest. There is increased temptation to deviate from the ethical norms for research and publication. Hence, obligation to ethics and ethical issues are prerequisite to be generated among scientific community,teachers, postgraduate and graduate students and professionals engaged in sponsored or self-sponsored research about what constitutes "research misconduct" and "deviations," so that one can guard against these and follow the ethical norms while perusing research/ publication as well as while reviewing articles submitted for editorial review.

In relation to biomedical research and publication, it has several perspectives for which a researcher needs to be knowledgeable and sensitive. These are:(i) Research design: Careful approach to research design, data collection and interpretation.(ii) Confidentiality: Maintaining confidentiality of research subjects and personnel records.(iii) Acknowledgement: Always acknowledging the source; using scientific materials without acknowledging the source amounts to plagiarism.(iv) Knowledge advancement: Aim to advance knowledge and research and not one's career. Avoid temptation for duplicate publication of the same research in different journals or in different languages, without proper declaration to the effect.(v) Risk/benefit: Protect the subjects/patients included in the research study, minimizing risks and maximizing benefits, specially the vulnerable population. Informed consent of the patient/guardian (in the case of minors) in the presence of witness is absolutely essential to protect the interests of the human subjects enrolled in the study.(vi) Animal design: Design animal experiments only if absolutely necessary and meaningful. Show proper care and compassion and minimize pain and suffering during the experiments.

\section{PRINCIPLES OF WRITING A SCIENTIFIC PAPER}

Scientific research demands precision. Scientific writing should respect this precision in the form of clarity. The scientific way of thinking and application of scientific methods require honesty, criticality, trust, creativity and openness. Acceptance of these principles are desirable 
prerequisites for successful engagement in science by students and young researchers. These help qualifying research institution that produces competent promoters (initiators) for the future technological cultural and political development of society. Defining principles of good scientific and good laboratory practice should encourage the development of standardized principles and guidelines for accurate and quality data in scientific research.

The text of observational and experimental articles: is usually divided into sections in accordance with socalled "IMRAD" structure: Introduction, Methods, Results, and Discussion. Papers related to public health programs and practice might have different than IMRAD structure (drug). There is a key question for each section of the IMRAD structure of the paper, which an author needs to keep in mind, while writing the manuscript.

Title of the scientific paper: contains a brief description of the content. The title should accurately describe the content of the article. There are two types of titles: Indicative title talks about the work that covers and informative title-convey the message of the article and recommended for beginners. A good title should be: (a) Short, (b) correct, (c) clear, (d) complete, (e) informing, (d) attractive. It should also include: Characteristics of the article, showing what is most important in the work. It is necessary to specify the names of the authors and their affiliations.

Abstract/summary and title can be written in two forms: Reference and Information. It can be written in author's native language and English.

The structure of the summary should look like this: Introduction, goal, materials and methods, the location of the study, measuring the outcomes of the study, the results and conclusions.

Summary: Summary is the distillate of which will be presented and should show: What has been done, what are the results, what the results means.

Introduction: Writing an introduction has its own rules: A clear definition of a the problem, why exactly this issue was explored, there is no need to explain what can be found in the textbooks, do not need to explain the terms of the title.

Materials and Methods: Materials and Methods describe how the study was conducted and the characteristics of the sample (experimental group, controls, and their properties). It is necessary to explain what is researched, asked, tested as follows: Sampling (random, consecutive, and representative), the sample size (patient gender, age), the control group, and the criteria for exclusion from the study, the control group if any.It should be described how the research was done: Type of study (prospective, retrospective or combined), data collection (surveys, inventory or checkup), the technique of measuring results (operative treatment, laboratory tests). It is necessary to specify where the research was conducted. .

Result: Results are an important part of writing an article. The research results are usually most carefully read and should be a detailed plan, well-documented

Discussion: Discussion is a critical review of the data described in the results. The results should be compared with other findings and discuss the theoretical and practical research outcome. It should point out gaps with explanation.

Conclusion: Conclusion should be short, clear and precise. It is necessary to make the final statement of what logically follows from the results of the work, list only the most important and give the message. Good conclusions should not surprise attentive reader. The reader should get the impression that he himself had written it.

References: References should be in accordance with the instructions provided by the journal, and otherwise used Vancouver or Harvard citation style.

ETHICAL PRINCIPLES FOR MEDICAL RESEARCH INVOLVING HUMAN SUBJECTS

The World Medical Association (WMA) has developed the Declaration of Helsinki \{http://www. hopitalmontfort.com/en/new-edition-declarationhelsinki1] as a statement of ethical principles for medical research involving human subjects, including research on identifiable human material and data. Consistent with the mandate of the WMA, the declaration is addressed primarily to physicians.

\section{General principles}

The Declaration of Geneva of the WMA binds the physician with the words, "The health of my patient will be my first consideration", and the International Code of Medical Ethics declares that, "A physician shall act 
in the patient's best interest when providing medical care".

Medical progress is based on research that ultimately must include studies involving human subjects. Medical research is subject to ethical standards that promote and ensure respect for all human subjects and protect their health and rights. It is the duty of physicians who are involved in medical research to protect the life, health, dignity, integrity, right to self-determination, privacy, and confidentiality of personal information of research subjects.

Physicians must consider the ethical, legal and regulatory norms and standards for research involving human subjects in their own countries as well as applicable international norms and standards. Medical research involving human subjects must be conducted only by individuals with the appropriate ethics and scientific education, training and qualifications.

Research registration and publication and dissemination of results

Every research study involving human subjects must be registered in a publicly accessible database before recruitment of the first subject. Researchers, authors, sponsors, editors and publishers all have ethical obligations with regard to the publication and dissemination of the results of research. Sources of funding, institutional affiliations and conflicts of interest must be declared in the publication. Reports of research not in accordance with the principles of this declaration should not be accepted for publication.

\section{PUBLICATION ETHICS}

Academic publishing depends, to a great extent, on trust. Editors trust peer reviewers to provide fair assessments, authors trust editors to select appropriate peer reviewers, and readers put their trust in the peer-review process.

Academic publishing also occurs in an environment of powerful intellectual, financial, and sometimes political interests that may collide or compete. Good decisions and strong editorial processes designed to manage these interests will foster a sustainable and efficient publishing system, which will benefit academic societies, journal editors, authors, research funders, readers, and publishers.Good publication practices do not develop by chance, and will become established only if they are actively promoted. The general principles of publication ethics are:

\section{Transparency}

Sources of funding for research or publication should always be disclosed. Editors should state this directly in their editorial policy. Authors should routinely include information about research funding in all papers they prepare for publication. Where a clinical trial registration number is available, this should be included.

\section{Authorship acknowledgment}

The International Committee of Medical Journal Editors (ICMJE) provides a definition of authorship that is applicable beyond the medical sector. The ICMJE authorship criteria state 'Authorship:

National institute of health (NIH) in its "Guidelines for Conduct of Research" have recommended that 1 . (Authorship): What is authorship?

In the literary world, an author is someone who creates a written work. In the academic research world, however, an author is much more

According to the Uniform Requirements for Manuscripts Submitted to Biomedical Journals, . (promoted by the International Committee of Medical Journal Editors (ICMJE).

Designation as an author must satisfy four conditions.

Author: The author must have:

1. Contributed substantially to the conception and design of the study, the acquisition of data, or the analysis and interpretation

2. Drafted or provided critical revision of the article

3. Provided final approval of the version to publish

4. Agreed to be accountable for all aspects of the work in ensuring that questions related to the accuracy or integrity of any part of the work are appropriately investigated and resolved

Authors of research papers should state whether they had complete access to the study data that support the publication. Contributors who do not qualify as authors should also be listed and their particular contribution described. This information should appear as an acknowledgment. Sample authorship description/ acknowledgment. Collecting authorship information for research papers, authorship should be decided at the study launch. Policing authorship is beyond the responsibilities of an editor. Editors should demand transparent and complete descriptions of who has contributed to a paper. 
Editors should employ appropriate systems to inform contributors about authorship criteria (if used) and/or to obtain accurate information about individuals' contributions.

Editors should ask authors to submit, as part of their initial submission package, a statement that all individuals listed as authors meet the appropriate authorship criteria, that nobody who qualifies for authorship has been omitted from the list, and that contributors and their funding sources have been properly acknowledged, and that authors and contributors have approved the acknowledgment of their contribution.

Manuscripts authored by a large group would need to list not only the name of involved organizations but also the members who satisfy the four authorship criteria listed above.

2. Acknowledgement: Those who do not meet these criteria but have still helped in some way to the study like providing technical help, writing assistance, or Department/Institutional head for providing access to the institutional infrastructure for the conduct of the research study, etc. should be relegated to the acknowledgement section.

If someone does not meet all four criteria to be named as an author, the ICMJE recommends acknowledgment credit instead of authorship

3.Authors/Contributor: An approach suggested to resolve the conflict between credit and responsibility for any research study is to designate "authors" as "contributors," each required to list their specific role in the study .

**4.Guarantor:one of the contributor/author may take up "Guarantorship," i.e., accountability for veracity of data and ethical conduct of all aspects of the study. (Many reputed international journals like JAMA, Nature, BMJ, etc. have adopted this approach.)

Honorary authorship is sometimes granted to those who played no significant role in the work, for a variety of reasons. The United States National Academy of Sciences, however, warns that such practices "dilute the credit due the people who actually did the work, inflates the credentials of those so 'honored,' and make the proper attribution of credit more difficult
A possible measure against honorary authorships has been implemented by some scientific journals, in particular by the Nature journals. They demand that each new manuscript must include a statement of responsibility that specifies the contribution of every author.

Ghost authorship occurs when an individual makes a substantial contribution to the research or the writing of the report, but is not listed as an author. Two-thirds of industry-initiated randomized trials may have evidence of ghost authorship.

Anonymous/unclaimed authorship:Authors occasionally forgo claiming authorship, for a number of reasons. Historically some authors have published anonymously to shield themselves when presenting controversial claims

Authorship order: Rules for the order of multiple authors in a list have historically varied significantly between fields of research. Some fields list authors in order of their degree of involvement in the work, with the most active contributors listed first; other fields, such as mathematics or engineering (e.g., control theory), sometimes list them alphabetically.Historically biologists tended to place a principal investigator (supervisor or lab head) last in an author list The following are some notable implications regarding author order.

- The "first author" is a coveted position because of the increased visibility., readers may falsely associate the first author with someone having more importance.

- Traditionally, the last author position is reserved for the supervisor or principal investigator. As such, this person receives much of the credit when the research goes well and the flak when things go wrong. The last author may also be the corresponding author, the person who is the primary contact for journal editors.

- Given that there is no uniform rule about author order, readers may find it difficult to assess the nature of an author's contribution to a research project.

- To address this issue, some journals, particularly medical ones, insist on detailed author contribution notes. Nevertheless, even this tactic does little to counter how strongly citation rules have enhanced the attention first-named authors receive. 


\section{Common methods for listing authors}

The following are some common methods for establishing author order lists.

1. Relative contribution. As mentioned above, the most common way authors are listed is by relative contribution. The author who most substantially worked on the draft article and the underlying research becomes the first author. The others are ranked in descending order of contribution. However, in many disciplines, such as the life sciences, the last author in a group is the principle investigator - the person who supervised the work.

2. Alphabetical list. Certain fields, particularly those involving large group projects, employ other methods. For example, high-energy particle physics teams list authors alphabetically.

3. Multiple "first" authors. Additional "first" authors can be noted by an asterisk or other symbol accompanied by an explanatory note. This practice is common in interdisciplinary studies; however, , the first name listed on a paper will still enjoy more visibility than any other "first" author.

4. Multiple "last" authors. Similar to recognizing several first authors, multiple last authors can be recognized via typographical symbols and footnotes. This practice arose as some journals wanted to increase accountability by requiring senior lab members to review all data and interpretations produced in their labs.

5. Negotiated order.. While there are clearer practices for designating first and last authors, there's no overriding convention for the middle authors. The list can be decided by negotiation, so sharpen those persuasive argument skills!

As it is evident that choosing the author order can be quite complicated; therefore, researchers should consider these factors early in the research process. Don't wait until the manuscript is drafted before you decide on the author order.

Responsibilities of Author: All authors, including coauthors, are usually expected to have made reasonable attempts to check findings submitted for publication. In some cases, co-authors of faked research have been accused of inappropriate behavior or research misconduct for failing to verify reports authored by others or by a commercial sponsor. Additionally, authors are expected to keep all study data for later examination even after publication.

\section{Attributing authorship to a group}

The ICMJE provides guidance for instances where a number of authors report on behalf of a larger group of investigators. This guidance is applicable outside the medical sector.

International Committee of Medical Journal Editors guidance states: "When a large, multi-center group has conducted the work, the group should identify the individuals who accept direct responsibility for the manuscript. These individuals should fully meet the criteria for authorship defined above... When submitting a group author manuscript, the corresponding author should clearly indicate the preferred citation and should clearly identify all individual authors as well as the group name". The individual authors who accept direct responsibility for the manuscript should list the members of the larger authorship group in an appendix to their acknowledgment.

\section{A REVIEWER}

It is the professional obligation and essential duty toward science to accept and to review the manuscript pertaining to one's discipline. It is an honor and a privilege to be asked to review a manuscript and should not be taken as a burden.

Reviewing involves evaluating the (i) scientific content and quality of research, (ii) clarity and logic of presentation, and (iii) ethical validity of the study.

It is not only the researcher but also the reviewer who should adhere to high ethical standards. The reviewer should provide accurate, courteous, unbiased, and justifiable report on the submitted manuscript in a timely manner. It is unbecoming of a reviewer to make derogatory comments and personal attacks in review of author's submission and rejecting a manuscript for publication even without reading it. Still worse, some unethical, unscrupulous reviewer can even try to block the publication and steal the idea and then take credit by publishing it themselves.

To guard against these possibilities, most journals send the manuscript to 2 or 3 reviewers. It is also expected that the manuscript copy is not retained and the data or its interpretation is not used before the publication of 
manuscript, without prior permission of the authors. However, the most important fact is that the reviewer has highest standard of integrity and ethical principles.

Peer review.: is the evaluation of work by one or more people of similar competence to the producers of the work (peers). It constitutes a form of self-regulation by qualified members of a profession within the relevant field

Professional peer review focuses on the performance of professionals, with a view to improving quality, upholding standards, or providing certification.

Professional peer review is common in the field of health care, where it is usually called clinical peer review

Scholarly peer review (also known as refereeing) is the process of subjecting an author's scholarly work, research, or ideas to the scrutiny of others who are experts in the same field, before a paper describing this work is published in a journal or as a book. The peer review helps the publisher (that is, the editor-in-chief or the editorial board) decide whether the work should be accepted, considered acceptable with revisions, or rejected.

Medical peer review may be distinguished in 4 classifications: 1) clinical peer review; 2) peer evaluation of clinical teaching skills for both physicians and nurses; ${ }^{[17][18]} 3$ ) scientific peer review of journal articles; 4) a secondary round of peer review for the clinical value of articles concurrently published in medical journals. ${ }^{[19]}$

\section{Ethical Guidelines for Reviewers}

1. Reviews should be objective evaluations of the research. If you cannot judge a paper impartially, you should not accept it for review or you should notify the editor as soon as you appreciate the Reviews should be constructive and courteous and the reviewer should respect the intellectual independence of the author. The reviewer should avoid personal comments; Science reserves the right to edit out comments that will hinder constructive discussion of manuscripts.

2. Just as you wish prompt evaluations of your own research, please return your reviews within the time period specified when you were asked to review the paper. If events will prevent a timely review, it is your responsibility to inform the editor at the time of the request.
3. The review process is conducted anonymously; Science never reveals the identity of reviewers to authors. The submitted manuscript is a privileged communication and must be treated as a confidential document. Please destroy all copies of the manuscript after review. Please do not share the manuscript with any colleagues without the explicit permission of the editor. Reviewers should not make personal or professional use of the data or interpretations before publication without the authors' specific permission (unless you are writing an editorial or commentary to accompany the article).

4. You should be aware of Science's policies for authors regarding conflict of interest, data availability, and materials sharing.

\section{Protecting research subjects}

Journals should ask authors to state that the study they are submitting was approved by the relevant Research Ethics Committee or Institutional Review Board. If human participants were involved, manuscripts must be accompanied by a statement that the experiments were undertaken with the understanding and appropriate informed consent of each.

Editors should reserve the right to reject papers if there is doubt whether appropriate procedures have been followed. If a paper has been submitted from a country where there is no Ethics Committee, Institutional Review Board, or similar review and approval, editors should use their own experience to judge whether the paper should be published. If the decision is made to publish a paper under these circumstances a short statement should be included to explain the situation.

Research misconduct: Not following the above ethical norms and scholarly conduct with an intent to deceive is termed "research misconduct."

\section{THE MAIN FORMS OF SCIENTIFIC AND PUBLISHING MISCONDUCT}

The Oxford English Dictionary describes fraud as "wrongful or criminal deception intended to result in financial or personal gain" and deceit as "the action or practice of deceiving someone by concealing or misrepresenting the truth".

Research organizations and the literature have defined these behavioral patterns within the umbrella title of 
"Research Misconduct".

There are three major and most severe forms of scientific fraud, scientific and publishing dishonesty or misconduct, in proposing, conducting or evaluation of research and presentation of the research results:

- Inventing data and results (fabrication);

- Alteration or changing the results (falsification); and

- Plagiarism (plagiarism), including self-plagiarism (self-plagiarism), fragmented, repetitive and double publication (duplicate publication).

In the process of publishing scientific papers, it is important to know how a completed research should be described in a scientific paper

Fabrication: Fabrication is the practice of inventing data or results and recording and/or reporting them in the research record.

Falsification : Falsification is the practice of omitting or altering research materials, equipment, data, or processes in such a way that the results of the research are no longer accurately reflected in the research record

Both of these schemes are probably among the most serious offenses in scientific research as they challenge the credibility of everyone and everything involved in a research effort.

Plagiarism: The term plagiarism stems from the Latin word plagium, meaning kidnapping a man. It literary means theft, taking material authored by others and presenting as someone else' Plagiarism is basically intended to deceive the reader's.Is appropriation of another person's idea, processes, results, text, or illustration/clinical photographs, etc. without its acknowledgement. Referring to the United States'Office of Research Integrity (ORI) definition of plagiarism, which is "unattributed textual copying.

Plagiarism can be divided into 1.direct (plagiarism of the text); 2.mosaic (the borrowing ideas and opinions from original source and a few verbatim words of phrases without crediting the author) and 3..selfplagiarism (which refers to re-using one's own work without citations).Plagiarism could also be termed as 4. "salami-slicing"-the selective use of research-project results to maximize the number of presentations possible- i.e., identical slices as described by Medial Journal Editors (MJE)(some does not recognize it a category).
Researchers rely on the published data, and have to be skilled to selectively process these data, to incorporate previous knowledge into a new paper, and to distinguish original ideas and research results from already publicized ones.

One more category in this is "Redundant (multiple) publication"- in which already published work is republished with new additional data

Journal instructions should clearly explain what is, and what is not, considered to be prior publication. Journals may choose to accept (i.e. consider "not redundant") the re-publication of materials that have been accurately translated from an original publication in a different language. Journals that translate and publish material that has been published elsewhere should ensure that they have appropriate permission ( $s$ ), should indicate clearly that the material has been translated and republished, and should indicate clearly the original source of the material. Editors may request copies of related publications if they are concerned about overlap and possible redundancy. Re-publishing in the same language as primary publication with the aim of serving different audiences is more difficult to justify when primary publication is electronic and therefore easily accessible, but if editors feel that this is appropriate they should follow the same steps as for translation. Editors should ensure that sub-group analyses, meta-and secondary analyses are clearly identified as analyses of data that have already been published, that they refer directly to the primary source, and that (if available) they include the clinical trial registration number from the primary publication.

So it may overemphasize the importance of the findings by its appearance more than once and can interfere with the process of metaanalysis, besides infringe on the International Copyright Law.

Research misconduct" are given as follows: (a)I ntentional or gross negligence leading to fabrication of scientific message or a false credit or emphasis given to a scientist. (b)Intentional distortion of the research process by fabrication of the text, hypothesis, or methods from another researcher's manuscript or publication or distortion of the research process in other ways.

Other deviation.research misconduct is unacceptable issue, other deviations" are activities that are considered unethical like. 
- Giving authorship to a colleague or relation who has not contributed substantially to the research study.

- Suppression" of unfavorable data which are in conflict with the researcher's or sponsor's (of the study) interest or an adverse event in a clinical research.

- Deliberate omission of contributions by other researchers to increase the importance of their own research study.

- Applying inappropriate statistical techniques to enhance the significance of one's own research.

- Deviating from the research protocol approved by the Institutional Ethical Committee.

- Not reporting an adverse event in a human research experiment.

It is important to understand why researchers indulge into scientific misconduct/ deviations. According to the "stressful" or "imperfect" environment theory, various institutional pressures, incentives, career ambitions, pursuit of fame, etc. can be the causes for misconduct. Poor supervision of junior researchers by teachers and guides is one of the contributing factors in scientific misconduct.

Conflict of interest:More generally conflict of interest can be defined as any situation in which an individual or corporation is in a position to exploit a professional or official capacity in some way for their personal or corporate benefit.

The board of ethics define "conflict of interest as a situation "in which personal and/or financial consideratios have potential to influence or compromise professional judgement in clinical service,research,consultation, instruction, administration or any other professional activity.

\section{INTERNATIONAL BODIES}

One of the oldest organizations dealing with research misconduct is the ORI in the United States. Set up in 1992, it oversees and directs Public Health Service research integrity activities. With a huge budget, it provides significant funds in the areas of health, research, and development, and oversees bodies such as The National Institute of Health and The Office of Public Health and Science.

The Committee on Publication Ethics (COPE) was established in 1997 by a small group of medical journal editors in the UK, but now has over 7000 members worldwide from all academic fields. Membership is open to editors of academic journals and others interested in publication ethics. Several major publishers (including Elsevier, Wiley-Blackwell, Springer, Taylor and Francis, Palgrave Macmillan and Wolters Kluwer) have signed up some, if not all, of their journals as COPE members. COPE provides advice to editors and publishers '. COPE does not investigate individual cases, but encourages editors to ensure that cases are investigated by the appropriate authorities (usually a research institution or employer).

The UK Research Integrity Office is another body representing the interests of over 50 universities and organizations dedicated to scientific research set up in 2006.

\section{CONCLUSIONS}

If one wants to create a scientific work, must have on his mind that creating a scientific work requires creativity and openness, honesty, trust, and obeying the ethical principles for writing a scientific paper.

As well an author in medical sciences should always follow the words; "The health of my patient will be my first consideration", (Declaration of Geneva, Adopted by the $2^{\text {nd }}$ General Assembly of the WMA, Geneva, Switzerland, September 1948).

While working on a an biomedical research involving human subjects medical workers should have on mind that it is the duty of the physician to remain the protector of the life and health of that person on whom biomedical research is being carried out. A researcher should know how to create a paper to publish a research work. He / she must know parts and pattern of a scientific paper. Follow the responsibilities of an author and obligation to ethics. 